\title{
On the Sum-capacity of the Linear Deterministic Interference Channel with Partial Feedback
}

\author{
Sy-Quoc Le ${ }^{1}$, Ravi Tandon ${ }^{2}$, Mehul Motani ${ }^{1}$, and H. Vincent Poor $^{2}$ \\ ${ }^{1}$ Department of ECE, National University of Singapore, Singapore. \\ ${ }^{2}$ Department of EE, Princeton University, Princeton, NJ.
}

\begin{abstract}
The linear deterministic interference channel (LDIC) with partial feedback is considered. Partial feedback for the LD-IC models a scenario in which the top $l$ most-significant-bits of the channel output of receiver $j$ are received as feedback at transmitter $j$, for $j=1,2$. The rationale for studying the LDIC with partial feedback comes from the fact that it is a good approximation to the Gaussian interference channel with output feedback corrupted by additive white Gaussian noise (commonly referred to as noisy feedback). The main contribution of this paper is a characterization of the sum-capacity of the symmetric LD-IC with partial feedback. The differences between the models of partial feedback and rate-limited feedback are emphasized and highlighted by comparing the corresponding sum-capacities, which are shown to differ in general.
\end{abstract}

\section{INTRODUCTION}

One of the most important issues for communication networks is that of interference management. Characterizing the capacity region of the two-user Gaussian interference channel (GIC) remains one of the fundamental unresolved problems in information theory. Recent breakthroughs in dealing with the capacity characterization of the GIC have made use of the linear deterministic interference channel (LD-IC) model [1], [2], [3]. The basic idea behind these works is that an appropriately defined LD model can serve as a good approximation to the Gaussian channel. By gaining valuable insights from studying the LD-IC, the proof techniques and ideas can be lifted over to the GIC. The capacity region of the GIC has been characterized to within 1-bit in [2].

It is well known that feedback does not increase the capacity of the discrete memoryless point-to-point channel. However, feedback does increase the capacity of multi-user channels. The fact that feedback increases the capacity of the discrete memoryless multiple-access channel (MAC) was shown by Gaarder and Wolf [4]. Afterwards, Ozarow [5] found the capacity region of the two-user Gaussian MAC with noiseless feedback. Recently, Suh and Tse [6] obtained an interesting result that noiseless feedback can provide significant capacity gains for the GIC. To understand the usefulness of feedback for the interference channel, consider the case of very-strong interference, in which the direct links are weaker than the cross (interference) links. In such a scenario, feedback can

The work of M. Motani and S.-Q. Le was supported in part by the National University of Singapore under Research Grant WBS R-263-000-579-112. Email: $\{$ le.sy.quoc, motani $\} @$ nus.edu.sg

The work of H. V. Poor and R. Tandon was supported in part by the Air Force Office of Scientific Research under MURI Grant FA9550-09-1-0643, and in part by the DTRA under Grant HDTRA-07-1-0037. E-mail: \{rtandon, poor\}@princeton.edu

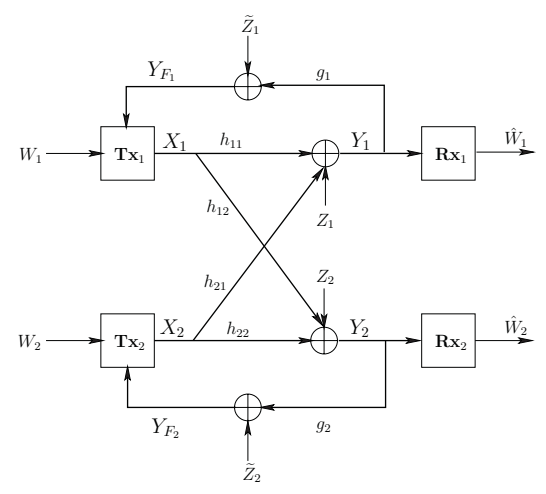

Fig. 1. The Gaussian interference channel (GIC) with noisy feedback.

provide a substantial capacity increment by utilizing the alternate path of $\mathrm{Tx}_{1} \rightarrow \mathrm{Rx}_{2} \rightarrow \mathrm{Tx}_{2} \rightarrow \mathrm{Rx}_{1}$, i.e., the information intended from $\mathrm{Tx}_{1}$ first reaches $\mathrm{Rx}_{2}$, which is then received as feedback at $\mathrm{Tx}_{2}$, which utilizes the strong cross (interference) link to reach the eventual destination at $\mathrm{Rx}_{1}$. The approximate capacity region of the GIC with noiseless channel output feedback has been characterized in [6] within 2-bits. The results in [6] have been generalized to the case of the fully connected $K$-user IC [7], and the case of the cyclic $K$-user IC [8]. Vahid et al. consider an interesting generalization of [6] by studying the two-user GIC with rate-limited feedback in [9]. Rate-limited feedback refers to a setting in which the receiver can utilize all the information it has received so far and feed back information over an orthogonal channel of finite capacity (i.e., a bit-pipe). Several interesting results for the GIC with rate-limited feedback are obtained in [9].

While rate-limited feedback may be useful in scenarios in which the feedback links have good coding schemes to protect feedback signals from error, it places much complexity on the receiver's side. As a result, this model is not appropriate when the complexity of the feedback design is a concern. In order to take this issue into account, this paper aims to investigate the model in which feedback signals are not well coded, and thus suffer from corruption by noise. In particular, if the channel output at receiver $j$ is $Y_{j}$, then the feedback to transmitter $j$ is $Y_{F, j}=g_{j} Y_{j}+\tilde{Z}_{j}$, for $j=1,2$ (see Figure 1). This allows us to investigate the trade-off between complexity and data rate. With the eventual goal of understanding the capacity region of the GIC with noisy feedback, we present a linear deterministic model with partial feedback. We show that the LD-IC with partial feedback serves as a good approximation to the GIC with noisy feedback. The main result of this paper 
is a complete characterization of the sum-capacity of the LDIC with partial feedback.

It is worth demarcating the model of partial feedback from the model of rate-limited feedback of [9], [10]. For the case of rate-limited feedback of $l$ bits, the receiver can exactly control (encode) which $l$ bits or functions thereof to feed back to its corresponding transmitter. In contrast, for the case of partial feedback, the receiver has no control over the feedback bits since the top $l$ most significant bits are received as feedback. Hence partial feedback can also be interpreted as naturally selected limited feedback. It is hence clear that the sumcapacity with $l$-bits of rate-limited feedback always serves as an upper bound on the sum-capacity with $l$-bits of partialfeedback. Our characterization of the sum-capacity of the LDIC with partial feedback reveals that these two models are markedly different, and the sum-capacity with rate-limited feedback can strictly exceed the sum-capacity with partial feedback.

\section{SYSTEM MODEL}

The two-user Gaussian interference channel with noisy feedback (see Figure 1), is defined by the following inputoutput relationships:

$$
\begin{aligned}
Y_{1 i} & =h_{11} X_{1 i}+h_{21} X_{2 i}+Z_{1 i} \\
Y_{2 i} & =h_{12} X_{1 i}+h_{22} X_{2 i}+Z_{2 i} \\
Y_{F_{1}, i} & =g_{1} Y_{1 i}+\tilde{Z}_{1 i} \\
Y_{F_{2}, i} & =g_{2} Y_{2 i}+\tilde{Z}_{2 i},
\end{aligned}
$$

where $X_{j i}$ denotes the signal sent by transmitter $j, Y_{j i}$ denotes the output at receiver $j$, and $Y_{F_{j}, i}$ denotes the feedback received at transmitter $j$, for $j=1,2$, at time $i$, for $i \in\{1,2, \ldots, T\}$, and $\left\{Z_{j i}\right\}_{i=1}^{T}$ and $\left\{\tilde{Z}_{j i}\right\}_{i=1}^{T}$ are independent, additive white Gaussian noise processes with zero means and unit variances. The forward channel gains $\left\{h_{11}, h_{21}, h_{12}, h_{22}\right\}$ and the feedback channel gains $\left\{g_{1}, g_{2}\right\}$ are assumed to be constant and known at all terminals. Average unit power constraints are imposed at each transmitter, i.e., for a code of block length $T$, input sequences must satisfy $\frac{1}{T} \mathbb{E}\left(\sum_{i=1}^{T}\left|X_{j i}\right|^{2}\right) \leq 1, j=1,2$. The transmitter $\mathrm{Tx}_{j}$, for $j=1,2$, wishes to communicate a message $m_{j} \in$ $\left\{1,2, \ldots, M_{j}\right\}=W_{j}$ to the receiver $\mathrm{Rx}_{j}$. It is assumed that $W_{1}$ and $W_{2}$ are independent. An $\left(M_{1}, M_{2}, T, P_{e}\right)$ feedback code for the interference channel (IC) with noisy feedback consists of a sequence of encoding functions

$$
\begin{aligned}
& f_{j}^{i}: \mathcal{W}_{j} \times\left\{\mathcal{Y}_{F_{j} 1}, \mathcal{Y}_{F_{j} 2}, \ldots, \mathcal{Y}_{F_{j}, i-1}\right\} \rightarrow \mathcal{X}_{j i} \\
& \text { for } j=1,2 \text {, and } i=1,2, \ldots, T ;
\end{aligned}
$$

and two decoding functions

$$
d_{j T}:\left\{\mathcal{Y}_{j 1}, \mathcal{Y}_{j 2}, \ldots, \mathcal{Y}_{j T}\right\} \rightarrow \hat{\mathcal{W}}_{j} \text { for } j=1,2 ;
$$

such that $\max \left\{P_{e, 1 T}, P_{e, 2 T}\right\} \leq P_{e}$, where $P_{e, 1 T}$ and $P_{e, 2 T}$ denote the average decoding error probabilities, which are computed as $P_{e, j T}=\mathcal{E}\left[P\left(\hat{w}_{j} \neq w_{j} \mid\left(w_{1}, w_{2}\right)\right.\right.$ were sent $\left.)\right]$. A rate pair $\left(R_{1}, R_{2}\right)$ is achievable for the IC with noisy feedback if there exists an $\left(M_{1}, M_{2}, T, P_{e}\right)$-feedback code such that $P_{e} \rightarrow 0$ as $T \rightarrow \infty, \frac{\log \left(M_{1}\right)}{T} \leq R_{1}$ and $\frac{\log \left(M_{2}\right)}{T} \leq R_{2}$. The

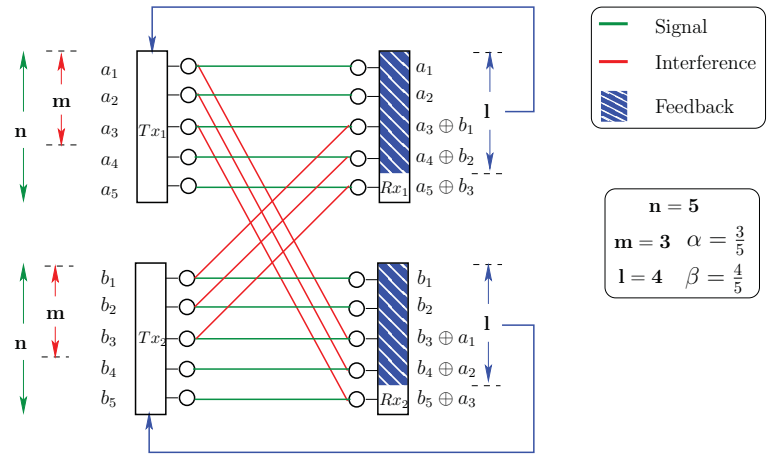

Fig. 2. The linear deterministic interference channel with partial feedback. capacity region of the IC with noisy feedback is defined as the closure of the set of all achievable rate pairs. With the goal of understanding the capacity region of the GIC with noisy feedback as defined above, we next describe the linear deterministic interference channel with partial feedback.

Using the deterministic model in [1], a non-negative integer $n_{k j}$ is used to represent the channel gain from the transmitter $\mathrm{Tx}_{k}$ to the receiver $\mathrm{Rx}_{j}$ and it is given by $n_{k j}=\left\lceil\log h_{k j}\right\rceil^{+}$. Let $q$ denote the maximum channel gains in the interference channel, i.e., $q=\sup \left(n_{k j}\right)$. Thus, the transmitted signal from the transmitter $k$ at the time $i$ will have a maximum of $q$ bits visible to any receiver. Denote $X_{k i}=\left[X_{k i}^{1}, \ldots, X_{k i}^{q}\right]^{T} \in F_{2}^{q}$, for $k=1,2$, where the leftmost bit is the most significant bit and the rightmost bit is the least significant bit. In this linear model, the effect of interference between various signals is captured as the superposition of those signals. At the time $i$, the outputs at the receivers are given as

$$
\begin{aligned}
& Y_{1 i}=S^{q-n_{11}} X_{1 i} \oplus S^{q-n_{21}} X_{2 i} \\
& Y_{2 i}=S^{q-n_{12}} X_{1 i} \oplus S^{q-n_{22}} X_{2 i},
\end{aligned}
$$

where $\mathrm{S}$ is the a square shift matrix of size $q$ and the operation is modulo 2 addition in $F_{2}$. Similarly, the channel gains for the feedback links can be represented by $l_{j}$, for $j=1,2$, where $l_{j}=\left\lceil\log \frac{g_{j}}{\sqrt{\operatorname{var}\left(Y_{j}\right)}}\right\rceil^{+}$, and hence the feedback signals at the transmitters are given as

$$
Y_{F_{1} i}=S^{q-l_{1}} Y_{1 i}, \quad Y_{F_{2} i}=S^{q-l_{2}} Y_{2 i} .
$$

Effectively, via the feedback links, the transmitter $j$ sees only the top $l_{j}$ bits of the received signals $R_{j i}$ (see Figure 2). The focus of this paper is on the symmetric LD-IC in which $m=n_{12}=n_{21}, n=n_{11}=n_{22}$, and $l=l_{1}=l_{2}$.

Definition 1. Given a triple $(n, m, l)$, let $\mathcal{C}^{\mathrm{P}-\mathrm{FB}}(n, m, l)$ denote the set of all achievable rate pairs $\left(R_{1}, R_{2}\right)$ with partial feedback. We define the sum-capacity with partial feedback as

$$
C_{\mathrm{sum}}^{\mathrm{P}-\mathrm{FB}}(n, m, l)=\sup _{\left(R_{1}, R_{2}\right) \in \mathcal{C}^{\mathrm{P}-\mathrm{FB}}(n, m, l)}\left(R_{1}+R_{2}\right) .
$$

We present our results in terms of forward and feedback interference parameters defined respectively as follows

$$
\alpha=\frac{m}{n}, \quad \beta=\frac{l}{n} .
$$


The forward interference parameter $\alpha$ measures the normalized interference ${ }^{1}$, whereas the feedback interference parameter $\beta$ measures the level of normalized feedback.

Definition 2. The normalized sum-capacity is given by

$$
C_{\mathrm{P}-\mathrm{FB}}^{*}(\alpha, \beta)=\frac{C_{\mathrm{sum}}^{\mathrm{P}-\mathrm{FB}}(n, m, l)}{n} .
$$

In a similar manner, we denote $C_{\mathrm{N}-\mathrm{FB}}^{*}(\alpha), C_{\mathrm{F}-\mathrm{FB}}^{*}(\alpha)$, and $C_{\mathrm{RL}-\mathrm{FB}}^{*}(\alpha, \beta)$ as the normalized sum-capacities without feedback, with full (perfect) feedback and with rate-limited feedback of $\beta$ (i.e., normalized rate-limited feedback of $(l / n)$ bits) respectively.

\section{MAIN RESUltS}

Theorem 1. The normalized sum-capacity of the symmetric LD-IC with partial feedback is given as follows

$$
\begin{aligned}
& C_{\mathrm{P}-\mathrm{FB}}^{*}(\alpha, \beta)= \\
& \begin{cases}\min \left((2-2 \alpha)+2(\beta-(1-\alpha))^{+}, 2-\alpha\right), & 0 \leq \alpha \leq \frac{1}{2} \\
\min \left(2 \alpha+2(\beta-\alpha)^{+}, 2-\alpha\right), & \frac{1}{2} \leq \alpha \leq \frac{2}{3} \\
2-\alpha, & \frac{2}{3} \leq \alpha \leq 1 \\
\alpha, & 1 \leq \alpha \leq 2 \\
\min \left(2+2(\beta-1)^{+}, \alpha\right), & 2 \leq \alpha .\end{cases}
\end{aligned}
$$

The detailed proof of Theorem 1 is omitted due to space limitations and can be found in [11]. In comparison, we recall here the corresponding (normalized) sum-capacities without feedback [1], [3], with full feedback [6], and with rate-limited feedback (of $l$-bits) [9]

$$
\begin{gathered}
C_{\mathrm{N}-\mathrm{FB}}^{*}(\alpha)= \begin{cases}2-2 \alpha, & 0 \leq \alpha \leq \frac{1}{2} \\
2 \alpha, & \frac{1}{2} \leq \alpha \leq \frac{2}{3} \\
2-\alpha, & \frac{2}{3} \leq \alpha \leq 1 \\
\alpha, & 1 \leq \alpha \leq 2 \\
2, & 2 \leq \alpha,\end{cases} \\
C_{\mathrm{F}-\mathrm{FB}}^{*}(\alpha)= \begin{cases}2-\alpha, & 0 \leq \alpha \leq 1 \\
\alpha, & 1 \leq \alpha,\end{cases} \\
C_{\mathrm{RL}-\mathrm{FB}}^{*}(\alpha, \beta)= \begin{cases}\min (2-2 \alpha+2 \beta, 2-\alpha), & 0 \leq \alpha \leq \frac{1}{2} \\
\min (2 \alpha+2 \beta, 2-\alpha), & \frac{1}{2} \leq \alpha \leq \frac{2}{3} \\
2-\alpha, & \frac{2}{3} \leq \alpha \leq 1 \\
\alpha, & 1 \leq \alpha \leq 2\end{cases} \\
\min (2+2 \beta, \alpha),
\end{gathered}
$$

We note here that these quantities satisfy the following inequalities

$$
\begin{aligned}
C_{\mathrm{N}-\mathrm{FB}}^{*}(\alpha) & \leq C_{\mathrm{P}-\mathrm{FB}}^{*}(\alpha, \beta) \\
& \leq C_{\mathrm{RL}-\mathrm{FB}}^{*}(\alpha, \beta) \leq C_{\mathrm{F}-\mathrm{FB}}^{*}(\alpha) .
\end{aligned}
$$

\footnotetext{
${ }^{1}$ with respect to $\mathrm{n}$
}

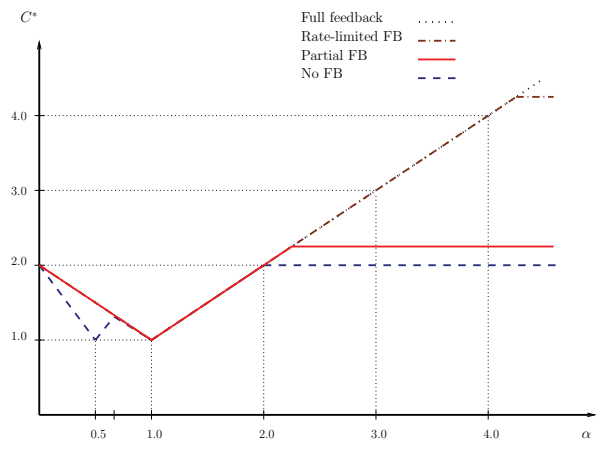

Fig. 3. Normalized sum-capacities for $\beta=\frac{9}{8}$.

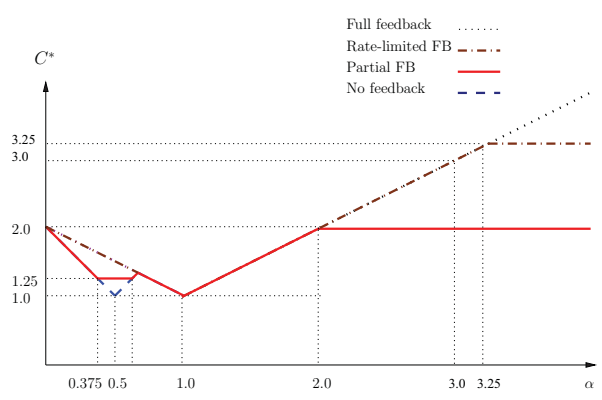

Fig. 4. Normalized sum-capacities for $\beta=\frac{5}{8}$.

Moreover, from Theorem 1, it is clear that all of the inequalities above can be strict inequalities in general, depending on the relative values of $(\alpha, \beta)$. As an example, we have shown all four sum-capacities as functions of $\alpha$, when $\beta=9 / 8$ in Figure 3; and when $\beta=5 / 8$ in Figure 4 . The effect of feedback on the sum-capacity of the partial feedback model differs from that of the rate-limited feedback model. The feedback helps to increase the sum rate only when the feedback rate is greater than a certain threshold depending on the specific regime. The threshold is found to be equal to half of the sum-capacities in the model of no feedback. Intuitively, there is a tradeoff between complexity and capacity here.

Case 1: Very weak interference: $0 \leq \alpha \leq \frac{1}{2}$

In this regime, feedback does not increase the sum-capacity $C_{\mathrm{P}-\mathrm{FB}}^{*}$ when $0 \leq \beta \leq 1-\alpha$. The sum-capacity starts to increase when $1-\alpha \leq \beta$. The difference between our normalized sum-capacity $C_{\mathrm{P}-\mathrm{FB}}^{*}$ and the sum-capacity in ratelimited feedback model $C_{\mathrm{RL}-\mathrm{FB}}^{*}$ is exactly $2(1-\alpha)$ in this case. The intuition behind this result is that the top $n(1-\alpha)$ bits of the feedback are received perfectly by the intended receiver. Thus, feedback is redundant when the feedback link is too small.

Case 2: Weak interference: $\frac{1}{2} \leq \alpha \leq \frac{2}{3}$

The result in this case differs from the previous case. In this regime, feedback does not increase the sum-capacity when $0 \leq \beta \leq \alpha$. The sum-capacity starts to increase when $\alpha \leq$ $\beta$. This result is counter-intuitive. Notice that only the top $n(1-\alpha)$ bits of the feedback are already received perfectly by the intended receiver. The result here reflects the nature of interference in this regime. The difference between $C_{\mathrm{P}-\mathrm{FB}}^{*}$ and $C_{\mathrm{RL}-\mathrm{FB}}^{*}$ is $2 \alpha$ in this regime.

Case 3: Moderate to strong interference: $\frac{2}{3} \leq \alpha \leq 2$ 


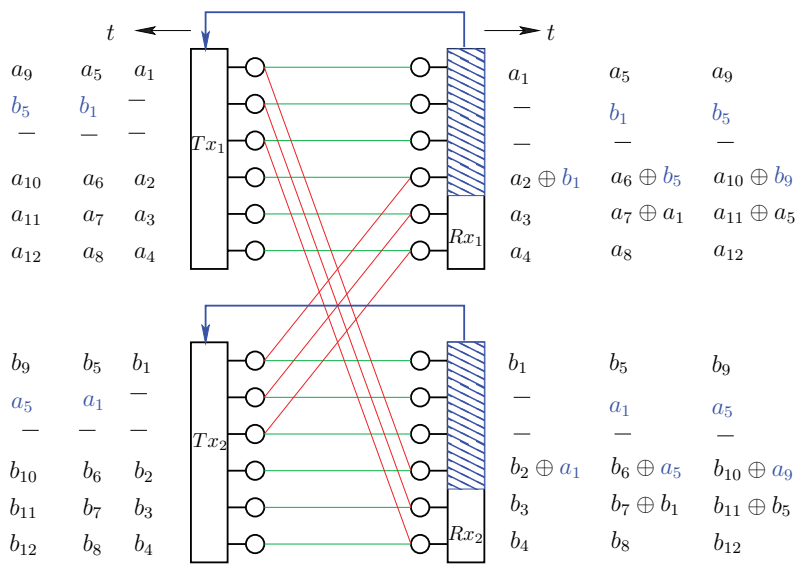

Fig. 5. Coding for the very weak interference regime: $(n, m, l)=(6,3,4)$.

Feedback does not increase the sum-capacity in this regime. Case 4: Very strong interference: $2 \leq \alpha$

In this regime, the sum-capacity starts to increase only when $1 \leq \beta$. Notice that this is the case of very strong interference, where all the bits in all positions are interfered by the interference channel. Feedback in our model helps only when the rate of feedback is greater than the direct link's. The difference between $C_{\mathrm{P}-\mathrm{FB}}^{*}$ and $C_{\mathrm{RL}-\mathrm{FB}}^{*}$ is 2 .

\section{CODING SCHEMES}

In this section, sum-capacity achieving coding schemes for specific values of $(n, m, l)$ are presented. Gaining intuition from these examples, encoding schemes for arbitrary $(n, m, l)$ are deduced in [11]. The converse is partially presented in the Appendix

\section{A. Very weak interference: $0 \leq \alpha \leq \frac{1}{2}$}

In this subsection, we present a capacity achieving encoding scheme for $n=6, m=3, l=4$, to build intuition for the general case. Based on the example, a general encoding scheme can be deduced (see Figure 5). From the previous section, it is clear that the sum-capacity with no feedback is 6 , with full feedback is 9 , and with rate-limited feedback (of 4 bits) is also 9 . Whereas, the sum-capacity with partial feedback of $l=4$ bits is 8 bits/channel use. To this end, we will show that it is possible to achieve the rate pair $\left(R_{1}, R_{2}\right)=(4,4)$ for the LD-IC in consideration.

In the first time slot $t=1$, each transmitter sends 4 fresh information bits as shown in the figure. With a feedback channel gain $l=4, \mathrm{Tx}_{1}$ sees only the top 4 bits, which are $a_{1},-,-, a_{2} \oplus b_{1}$, and hence it can recover $b_{1}$. Notice that the feedback signal $a_{1}$ is redundant since it is already received cleanly at the intended transmitter $\mathrm{Rx}_{1}$. This is the price we have to pay for partial feedback in contrast to ratelimited feedback. In the second time slot $i=2$, the transmitter $\mathrm{Tx}_{1}$ sends 4 new fresh information bits again and encodes $b_{1}$ at the second topmost signal level as shown in the figure. The second topmost signal level is chosen to ensure that the resolving signal bit $b_{1}$ is received cleanly at $\mathrm{Tx}_{1}$. With the help of $b_{1}, \mathrm{Rx}_{1}$ can resolve the interference in the previous time slot and decode $a_{2}$ successfully. Due to symmetry, the same encoding operation is carried out at $\mathrm{Tx}_{2}$ and $\mathrm{Rx}_{2}$.

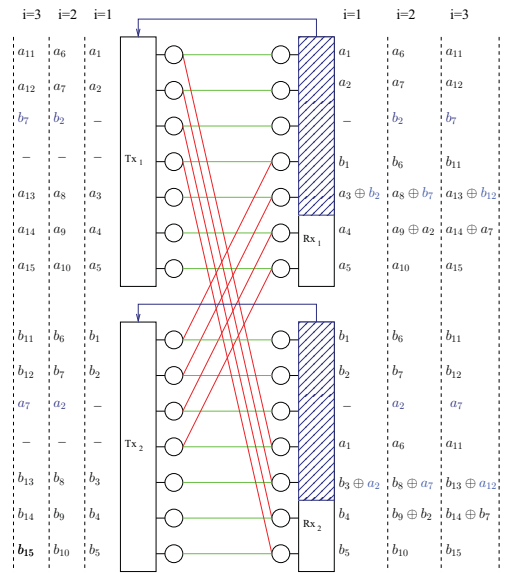

Fig. 6. Coding for the weak interference regime: $(n, m, l)=(7,4,5)$.

We can repeat this encoding scheme again for a duration of $B$ time slots. It is easy to see that this scheme asymptotically achieves a sum rate of 8 bits/channel use, which matches with the normalized sum rate of $2 \beta$ as stated in Theorem 1 .

\section{B. Weak interference: $\frac{1}{2} \leq \alpha \leq \frac{2}{3}$}

In this subsection, we consider the specific example, where $n=7, m=4$ and $l=5$. This corresponds to the case $\alpha \leq$ $\beta \leq 1$ (see Figure 6). From the figure, we can see that a sum rate of 10 bits/channel use, i.e., a normalized rate of $2 \alpha$ is asymptotically achievable, matching with the result in Theorem 1 .

\section{Very strong interference: $2 \leq \alpha$}

The interference channel with $n=1, m=6, l=2$, i.e., $\alpha=6, \beta=2$ is considered (see Figure 7). From the figure, it is easy to see that a sum rate of 4 bits/channel use, or a normalized sum rate of $2 \beta$ is achievable, matching the result in Theorem 1.

\section{Conclusions}

In this paper, we have characterized the sum-capacity region of the deterministic interference channel with partial feedback. In addition, our result has been analyzed and compared with three other related models: without feedback, with full feedback and with rate-limited feedback. Being different from the rate-limited model, the feedback in our model helps to improve the sum-capacity only when the channel gains for the feedback links exceed certain thresholds, depending on the specific regimes. As on-going work, we are focusing on the capacity region of the GIC with output feedback corrupted by additive white Gaussian noise.

\section{APPENDIX}

\section{A. Proof for very strong interference: $2 \leq \alpha$}

In this section, we present a sum-capacity achieving encoding scheme and a converse proof for the very strong interference regime $(2 \leq \alpha)$. The proof for the other regimes can be found in [11]. 


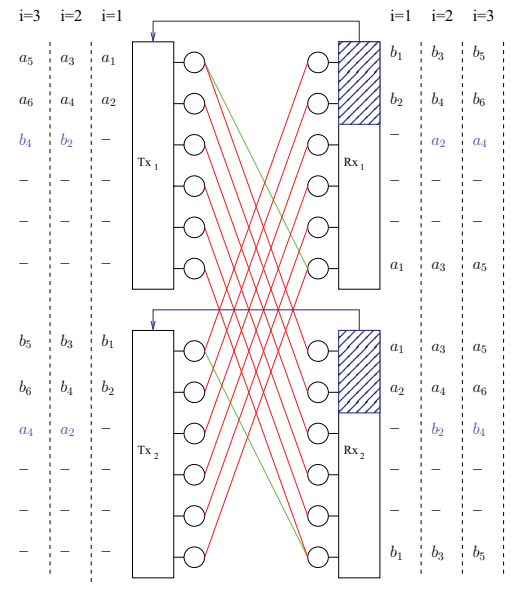

Fig. 7. Very strong interference: coding for $(n, m, l)=(1,6,2)$

1) Encoding Scheme for $(n, m, l)$ such that $2 n \leq m$.: For the case of strong interference, $\max (m, n)=m$. The set of $m$ bits at the transmitter $\mathrm{Tx}_{1}$ (respectively $\mathrm{Tx}_{2}$ ) is partitioned into 3 regions $A_{1}, A_{2}, A_{3}$, with sizes $(n, m-2 n, n)$. According to the partition sizes, the $m$ bits (channel output) received at $\mathrm{Rx}_{1}$ can be described by: $Y_{1}=\left(B_{1}, B_{2}, B_{3} \oplus A_{1}\right)$ (see Figure 8). From Theorem 1, it is clear that feedback increases sum-capacity only if $l>n$. Hence, we focus on the case in which $n<l$.

In all time instances, $\mathrm{Tx}_{1}$ encodes $n$ fresh information bits in $A_{1}$. An additional $E_{11}$ bits are encoded in the region $A_{2}$. The optimal size of $E_{11}$ is a function of $(l, m, n)$, which is chosen as $\min \left((l-n), \frac{m}{2}-n\right)$. It remains silent in region $A_{3}$ to avoid interference. Similarly, $\mathrm{Tx}_{2}$ sends fresh bits in $\left(B_{1}, E_{21}\right)$ and remains silent in $B_{3}$.

In this first time slot, $\mathrm{Rx}_{2}$ can receive $E_{11}$ cleanly; however $\mathrm{Rx}_{1}$ cannot. Moreover, via feedback of $l$ most significant bits, $\mathrm{Tx}_{2}$ has access to $E_{11}$ and $\mathrm{Tx}_{1}$ has access to $E_{21}$. In the second time slot, $\mathrm{Tx}_{1}$, besides sending fresh bits in the top $\min \left(l, \frac{m}{2}\right)$ levels, forwards the bits $E_{21}$ in the remaining part of $A_{2}$. Similarly, $\mathrm{Tx}_{2}$ forwards the bits $E_{11}$ in the remaining part of $B_{2}$. Thus, in this second time slot, $\mathrm{Rx}_{1}$ can receive $E_{11}$, and $\mathrm{Rx}_{2}$ can receive $E_{21}$. From the third time slot onwards, the same operation is repeated. A sum rate of $\min (2 l, m)$ bits/channel use, or a normalized rate of $\min (2 \beta, \alpha)$ is achievable.

2) Converse for $(n, m, l)$ such that $2 n \leq m_{.}:$Let $Y_{F_{j}}^{T}$ for $j=1,2$, denote the feedback from $\mathrm{Rx}_{j}$ to $\mathrm{Tx}_{j}$ in the duration of $T$ channel uses. Please refer to Figure 8 for the partition. We first state the following lemma, which gives an upper bound on $R_{1}$ and $R_{2}$.

Lemma 1. For $\alpha \geq 2$, we have $R_{j} \leq n+(l-n)^{+}$for $j=1,2$.

\section{Proof:}

$$
\begin{aligned}
& T R_{1}=H\left(W_{1}\right)={ }^{(a)} H\left(W_{1} \mid W_{2}\right) \\
& =I\left(W_{1} ; A_{1}^{T}, Y_{F_{2}}^{T} \mid W_{2}\right)+H\left(W_{1} \mid W_{2}, A_{1}^{T}, Y_{F_{2}}^{T}\right) \\
& ={ }^{(b)} I\left(W_{1} ; A_{1}^{T}, Y_{F_{2}}^{T} \mid W_{2}\right)+H\left(W_{1} \mid W_{2}, A_{1}^{T}, Y_{F_{2}}^{T}, X_{2}^{T}, Y_{1}^{T}\right) \\
& \leq H\left(A_{1}^{T}, Y_{F_{2}}^{T}\right)+H\left(W_{1} \mid Y_{1}^{T}\right)
\end{aligned}
$$

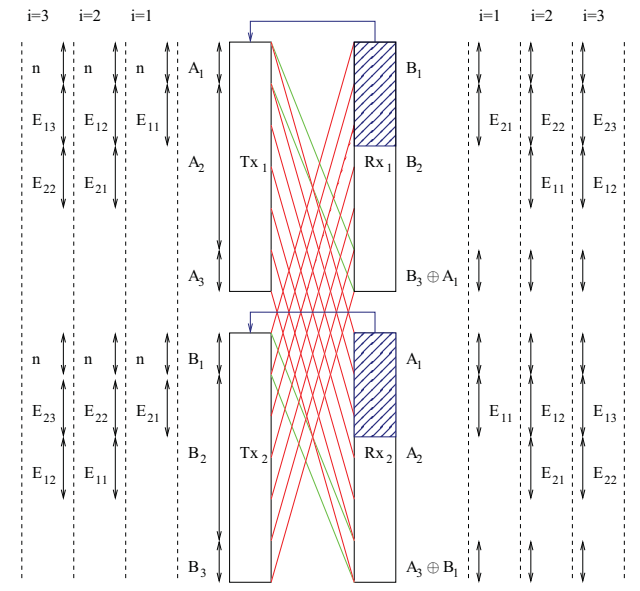

Fig. 8. Very strong interference: coding for arbitrary $(n, m, l)$.

$$
\leq^{(c)} H\left(A_{1}^{T}, Y_{F_{2}}^{T}\right)+1+T P_{e}^{T},
$$

where (a) follows from the independence between $W_{1}$ and $W_{2}$; (b) follows from the fact $X_{2}^{T}$ is a function of $\left(W_{2}, Y_{F_{2}}^{T}\right)$, and $Y_{1}^{T}$ is a function of $\left(A_{1}^{T}, X_{2}^{T}\right)$ and (c) follows from Fano's inequality. We next bound the term $H\left(A_{1}^{T}, Y_{F_{2}}^{T}\right)$.

Case 1: $0 \leq l \leq n$. For this case, $Y_{F_{2}}^{T}$ is a function of $A_{1}^{T}$ and we have $H\left(A_{1}^{T}\right) \leq n T$.

Case 2: $n \leq l \leq m$. In this case, $A_{1}^{T}$ is a function of $Y_{F_{2}}^{T}$. We have $H\left(Y_{F_{2}}^{T}\right) \leq l T$.

From both these cases, we conclude that $R_{1} \leq n+(l-n)^{+}$. The inequality for $R_{2}$ can be proved in a similar manner.

From Lemma 1 , we have $R_{1}+R_{2} \leq 2 n+2(l-n)^{+}$, thus proving the converse for the regime $\alpha \geq 2$.

\section{REFERENCES}

[1] S. Avestimehr, S. Diggavi, and D. N. C. Tse. Wireless network information flow: A deterministic approach. IEEE Transactions on Information Theory, 57(4):1872-1905, Apr. 2011.

[2] R. H. Etkin, D. N. C. Tse, and H. Wang. Gaussian interference channel capacity to within one bit. IEEE Transactions on Information Theory, 54:5534-5562, Dec. 2008

[3] G. Bresler and D. N. C. Tse. The two-user Gaussian interference channel: A deterministic view. European Transactions on Telecommunications, 19(4):333-354, Jun. 2008

[4] N.T. Gaarder and J. K. Wolf. The capacity region of a multipleaccess discrete memoryless channel can increase with feedback. IEEE Transactions on Information Theory, 21(1):100-102, Jan. 1975.

[5] L. H. Ozarow. The capacity of the white Gaussian multiple access channel with feedback. IEEE Transactions on Information Theory, 30(4):623-629, Jul. 1984

[6] C. Suh and D. N. C. Tse. Feedback capacity of the Gaussian interference channel to within 2 bits. IEEE Transactions on Information Theory, 57(5):2667-2685, May. 2011.

[7] S. Mohajer, R. Tandon, and H. V. Poor. On the feedback capacity of the fully connected $K$-user interference channel. IEEE Transactions on Information Theory, submitted 2011.

[8] R. Tandon, S. Mohajer, and H. V. Poor. On the symmetric feedback capacity of the $K$-user cyclic Z-interference channel. IEEE Transactions on Information Theory, submitted 2011.

[9] A. Vahid, C. Suh, and S. Avestimehr. Interference channel with ratelimited feedback. IEEE Transactions on Information Theory, 58:27882812, May. 2012.

[10] A. Vahid and A. S. Avestimehr. The two-user deterministic interference channel with rate-limited feedback. In Proceedings of IEEE Int. Symp. Inf. The., pages 460-464, Austin, TX, 2010.

[11] S.-Q. Le, R. Tandon, M. Motani, and H. V. Poor. On the sum-capacity of the linear deterministic interference channel with partial feedback. Technical report available at http://dl.dropbox.com/u/5998959/motani/pubs/LD-IC-PF.pdf, 2012. 